\title{
ON RINGS WHOSE LEFT MODULES ARE DIRECT SUMS OF FINITELY GENERATED MODULES
}

\author{
KENT R. FULLER ${ }^{1}$
}

\begin{abstract}
The relationship between rings of finite module type and rings whose left modules have decompositions that complement direct summands is examined by proving that the latter are precisely the rings of the title.
\end{abstract}

A ring $\Delta$ with identity is said to be of finite module (or representation) type in case it is left artinian and has only finitely many (isomorphism types of) finitely generated indecomposable left modules. Such a ring is also right artinian and has only finitely many (the same number) finitely generated indecomposable right modules (Eisenbud and Griffith [7]). Auslander [2], [3] and Ringel and Tachikawa [17] have proved that every module over a ring of finite module type is a direct sum of finitely generated modules, and Tachikawa [17] has shown that they all have decompositions $M=\bigoplus_{A} M_{\alpha}$ that complement direct summands in the sense [1] that for each direct summand $K$ of $M$ there is a subset $B \subseteq A$ with $M=K \oplus\left(\oplus_{B} M_{\beta}\right)$.

Chase [6] proved that the rings of the title are left artinian. (See also [8] and [11].) More recently, Auslander [5] has proved that if $\Delta$ is finitely generated over its center and each left $\Delta$-module is a direct sum of finitely generated modules, then $\Delta$ is of finite module type, and Fuller and Reiten [9] have noted that if every left and every right $\Delta$-module has a decomposition that complements direct summands, then $\Delta$ is of finite module type. However, it is still not known whether either of these conditions on its left modules alone forces an arbitrary ring $\Delta$ with identity to be of finite module type. Our purpose here is to prove that nevertheless they are equivalent, and to show how their satisfaction depends on the structure of the finitely generated indecomposable left $\Delta$-modules.

We say that a ring $R$ has enough idempotents in case there exists orthogonal idempotents $\left(e_{\alpha}\right)_{\alpha \in A}$ in $R$ (called a complete set of idempotents for $R$ ) such that $R=\oplus_{A} R e_{\alpha}=\oplus_{A} e_{\alpha} R$. By an $R$-module we mean an $R$-module with a spanning set; so " ${ }_{R} M$ is a left $R$-module" implies $M=R M=\oplus_{A} e_{\alpha} M$. (Note then that if $1 \in R$, an $R$-module is just a unital one.) We denote the categories of left and right $R$-modules by ${ }_{R} \Re$ and $\Re_{R}$, and mention that they contain the regular modules ${ }_{R} R$ and $R_{R}$, respectively, and all ordinary submodules of their objects.

Received by the editors April 8, 1974 and, in revised form, November 14, 1974.

AMS (MOS) subject classifications (1970). Primary 16A46, 16A48; Secondary 16A40.

Key words and phrases. Finite module type, decomposition that complements direct summands.

${ }^{1}$ Author's research supported by NSF Grant GP-18828. 
Harada [12] and [13] has pointed out that over a ring $R$ with enough idempotents ordinary direct sums and tensor products behave as they do over a ring with identity, and that the projective $R$-modules are the direct summands of free modules. Moreover, he has observed that Bass's Theorem $P$ is valid for $R$. I.e., the following are equivalent: (a) every left $R$-module has a projective cover; (b) $R$ has a complete set of local idempotents and $J(R)$ is left $T$-nilpotent; (c) every flat left $R$-module is projective; (d) $R$ has d.c.c. on principal right ideals.

There is a slight difference for direct products though. The (categorical) direct product of $\left(M_{\gamma}\right)_{\gamma \in C}$ in ${ }_{R}$ N is not the ordinary cartesian product $\prod_{C} M_{\gamma}$, but rather $R \cdot\left(\Pi_{C} M_{\gamma}\right)$, which we denote by $\hat{\Pi}_{C} M_{\gamma}$. It is easy to see that this is indeed a direct product in $R^{\mathscr{T}}$, and, using the idempotents $\left(e_{\alpha}\right)_{\alpha \in A}$, that

$$
\begin{aligned}
\prod_{C} M_{\gamma}= & \left\{r x \in \prod_{C} M_{\gamma} \mid r \in R, x \in \prod_{C} M_{\gamma}\right\} \\
& =\left\{x \in \prod_{C} M_{\gamma} \mid x=e x \text { for some } e=e^{2} \in R\right\} .
\end{aligned}
$$

Of course, $\oplus_{C} M_{\gamma} \leqslant \hat{\Pi}_{C} M_{\gamma}$ and the usual injections and projections do their respective jobs. We also write $M^{(C)}$ for the direct sum of card $C$ copies of $M$, $M \stackrel{C}{C}$ for the direct product in $R^{\Re}$, and $M^{C}$ for the cartesian product.

We shall write all homomorphisms on the right, so $f g$ means "first $f$ then $g$ ".

The principal tool that we shall use is a particular ring with enough idempotents. Let $\Delta$ be a ring with identity and let ${ }_{\Delta} U=\oplus_{A} U_{\alpha}$ be a direct sum of finitely generated submodules $\left(U_{\alpha}\right)_{\alpha \in A}$. Let

$$
R=\left\{r:{ }_{\Delta} U \rightarrow{ }_{\Delta} U \mid U_{\alpha} r=0, \text { a.e. }\right\}
$$

("a.e." means "for all but finitely many $\alpha \in A$ "), and let $\left(e_{\alpha}\right)_{a \in A}$ be the orthogonal set of idempotents in $R$ such that $U e_{\alpha}=U_{\alpha}(\alpha \in A)$. We call $R$ the functor ring of the $\left(U_{\alpha}\right)_{\alpha \in A}$, because, although it does not really concern us here, the category ${ }_{R} \mathfrak{N}$ (resp., $\mathfrak{R}_{R}$ ) is equivalent to the category of contravariant (covariant) additive functors from the full subcategory $\Delta_{\Delta} \mathscr{U}$ of $\Delta^{\mathfrak{N}}$ with objects $\left\{U_{\alpha} \mid \alpha \in A\right\}$ to the category of abelian groups (e.g., see [10]). In particular, if $\left(U_{\alpha}\right)_{\alpha \in A}$ consists of one isomorphic copy of each finitely generated left $\Delta$-module, $R$ is the functor ring of the finitely generated left $\Delta$ modules.

Let ${ }_{\Delta} U=\oplus_{A} U_{\alpha}$ (with each $U_{\alpha}$ finitely generated) and $R$ be as above. Then ${ }_{\Delta} U_{R}$ is a bimodule and, letting

$$
\overline{\operatorname{Hom}}_{\Delta}(U, M)=\left\{\varphi:{ }_{\Delta} U \rightarrow{ }_{\Delta} M \mid U_{\alpha} \varphi=0, \text { a.e. }\right\}
$$

and, for each $f:{ }_{\Delta} M \rightarrow{ }_{\Delta} M^{\prime}$ in ${ }_{R} \mathfrak{T h}$

$$
f_{*}=\overline{\operatorname{Hom}}_{\Delta}(U, f): \varphi \mapsto \varphi f \quad(\varphi \in \overline{\operatorname{Hom}}(U, M)),
$$

we obtain a covariant functor $\overline{\operatorname{Hom}}_{\Delta}(U,-): \Delta_{\Delta} \Re \rightarrow{ }_{R} \Re$ that commutes with direct products $\left(\overline{\operatorname{Hom}}_{\Delta}\left(U, \Pi_{C} M_{\gamma}\right) \cong \hat{\Pi}_{C} \overline{\operatorname{Hom}}_{\Delta}\left(U, M_{\gamma}\right)\right)$ and direct sums (since $\operatorname{Im} \varphi$ is finitely generated for all $\varphi \in \overline{\operatorname{Hom}}_{\Delta}(U, M)$ ), and has left adjoint $\left(U \otimes_{R}-\right):{ }_{R} \mathfrak{R} \rightarrow{ }_{\Delta} \mathfrak{R}$. There are natural transformations 


$$
\nu: U \otimes_{R} \overline{\operatorname{Hom}}_{\Delta}(U,-) \rightarrow 1_{\Delta}, \quad \eta: 1_{R} \Re \rightarrow \overline{\operatorname{Hom}}_{\Delta}\left(U,\left(U \oplus_{R}-\right)\right)
$$

defined via

$$
\nu_{M}: u \otimes \varphi \mapsto u \varphi, \quad n \eta_{N}: u \mapsto u \otimes n
$$

Moreover, since $\overline{\operatorname{Hom}}_{\Delta}(U, U)=R, \nu_{\Delta} U: U \otimes_{R} \overline{\operatorname{Hom}}_{\Delta}(U, U) \rightarrow{ }_{\Delta} U$ and $\eta_{R} R$ : $R \rightarrow \overline{\operatorname{Hom}}_{\Delta}\left(U,\left(U \otimes_{R} R\right)\right)$ are the canonical isomorphisms. Thus, since both functors commute with direct sums, they restrict to inverse category equivalences

$\overline{\operatorname{Hom}}_{\Delta}(U,-): \operatorname{Add}\left({ }_{\Delta} U\right) \rightarrow \operatorname{Proj}\left({ }_{R} R\right), \quad\left(U \otimes_{R}-\right): \operatorname{Proj}\left({ }_{R} R\right) \rightarrow \operatorname{Add}\left({ }_{\Delta} U\right)$

where $\operatorname{Add}\left({ }_{\Delta} U\right)$ and $\operatorname{Proj}\left({ }_{R} R\right)$ are, respectively, the full subcategories of ${ }_{\Delta} \mathfrak{T}$ and ${ }_{R} \Re$ whose objects are the direct summands of direct sums of copies of $U$ and the projective left $R$-modules.

In the terminology of Auslander [4], a family of homomorphisms is called noetherian in case, for each sequence of nonisomorphisms

$$
M_{0} \stackrel{f_{0}}{\longrightarrow} M_{1} \stackrel{f_{1}}{\longrightarrow} M_{2} \rightarrow \cdots
$$

in the family, there exists an $n$ such that $f_{0} f_{1} \cdots f_{n}=0$. The family is conoetherian if it satisfies the obvious dual condition. In [4] he proved that if, over an artinian ring $\Delta$, the families of monomorphisms and of epimorphisms between finitely generated indecomposable left modules are, respectively, noetherian and conoetherian, then $\Delta$ is of finite module type (cf. condition (d) in our Theorem below).

With the above notation and conventions, and acknowledging a great debt to the ideas of Auslander [3] and Harada [12], [13], we are now in position to prove the

THEOREM . Let $\Delta$ be a ring with identity and let $R$ be the functor ring of the finitely generated left $\Delta$-modules. Then the following are equivalent:

(a) Every left $\Delta$-module is a direct sum of finitely generated modules.

(b) Every direct product of projective left $R$-modules is projective.

(c) $R$ is left perfect.

(d) $1 \in \Delta$ is a sum of orthogonal primitive idempotents and the family of homomorphisms between finitely generated indecomposable left $\Delta$-modules is noetherian.

(e) Every left $\Delta$-module has a decomposition that complements direct summands.

Proof. (a) $\Rightarrow$ (b). Assume (a). Then $\operatorname{Add}\left({ }_{\Delta} U\right)={ }_{\Delta} \mathfrak{T}$, and $\operatorname{Proj}\left({ }_{R} R\right)$ $\approx \operatorname{Add}\left({ }_{\Delta} U\right)$ is closed under direct products.

(b) $\Rightarrow$ (c). For this implication, upon noting that for each $a \in R, a \cdot\left(R^{\hat{C}}\right)$ is just the ordinary abelian group direct product $(a R)^{C}$, one can virtually copy Chase's proof [6, Theorem 3.1] to show (b) implies that $R$ has d.c.c. on principal right ideals.

(c) $\Rightarrow$ (a). Let $M \in{ }_{\Delta} \mathfrak{T}$, let $C=\overline{\operatorname{Hom}}_{\Delta}(U, M)$ and let $f=\oplus_{C} \gamma: U^{(C)}$ $\rightarrow M$. Then, since ${ }_{\Delta} \Delta$ is isomorphic to one of the $U_{\alpha}$ (so ${ }_{\Delta} U$ is a generator), we 
have an epimorphism $U^{(C)} \stackrel{f}{\rightarrow} M \rightarrow 0$ such that each $\gamma \in \overline{\operatorname{Hom}}_{\Delta}(U, M)$ factors through $f$.

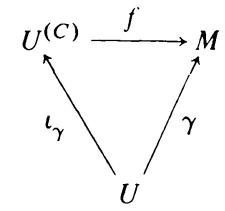

So, since here $\gamma=e \gamma=e$ ef for some $e=e^{2} \in R$, the sequence

$$
\overline{\operatorname{Hom}}_{\Delta}\left(U, U^{(C)}\right) \stackrel{f_{*}}{\longrightarrow} \overline{\operatorname{Hom}}_{\Delta}(U, M) \rightarrow 0
$$

is exact. But $\overline{\operatorname{Hom}}_{\Delta}(U, M)=\cup\left\{\overline{\operatorname{Hom}}_{\Delta}(U, L) \mid L \leqslant M\right.$ and $L$ is finitely generated $\}$ is a direct limit of projective left $R$-modules, so the hypothesis (c) implies that $f_{*}$ splits. Now, since ${ }_{\Delta} U$ is a generator implies that each $\nu_{M}$ is epic, we can apply the 5-lemma to

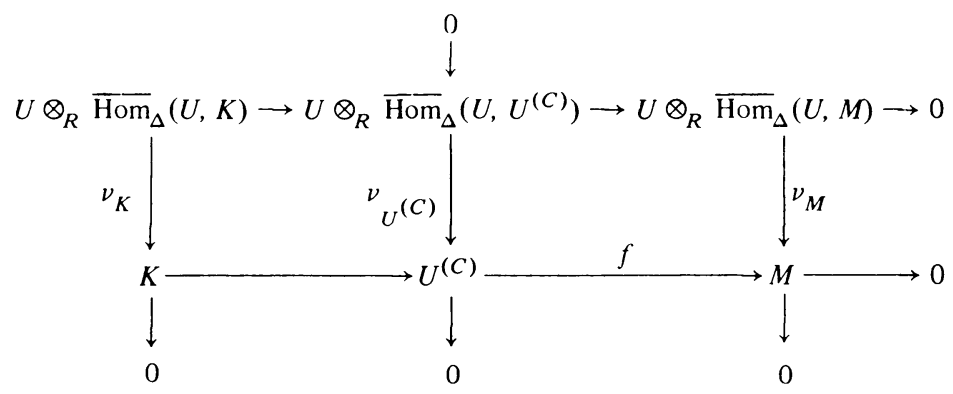

(with $K=\operatorname{Ker} f$ ) to see that $v_{M}$ is an isomorphism, and hence that $f$ also splits. Thus $\operatorname{Add}\left({ }_{\Delta} U\right)={ }_{\Delta} \mathfrak{R}$. Finally, since (c) also implies that each $\operatorname{End}\left({ }_{\Delta} U_{\alpha}\right)$ $\cong e_{\alpha} R e_{\alpha}$ is left perfect (so $U_{\alpha}=V_{\alpha 1} \oplus \cdots \oplus V_{\alpha n}$ with each $\operatorname{End}_{\Delta}\left(V_{\alpha i}\right)$ local), the Crawley-Jønsson-Warfield theorem [18] shows that every module in $\operatorname{Add}\left({ }_{\Delta} U\right)$ is a direct sum of finitely generated modules (with local endomorphism rings).

(c) $\Rightarrow(d)$. Assume (c) and suppose we have a sequence of $\Delta$-maps

$$
U_{\alpha_{0}} \stackrel{a_{0}}{\longrightarrow} U_{\alpha_{1}} \stackrel{a_{1}}{\longrightarrow} U_{\alpha_{2}} \rightarrow \cdots
$$

with no $a_{i}$ an isomorphism and each $U_{\alpha_{i}}$ indecomposable. Then $U_{\alpha_{i}}=U e_{\alpha_{i}}$ and $a_{i}=e_{\alpha_{i}} a_{i} e_{\alpha_{i+1}}$. Let $J=J(R)$. If $e_{\alpha_{i}}=e_{\alpha_{i+1}}$ then, since $e_{\alpha_{i}} R e_{\alpha_{i}}$ is local, $a_{i} \in J\left(e_{\alpha_{i}} R e_{\alpha_{i}}\right) \stackrel{\alpha_{i+1}}{=} e_{\alpha_{i}} J e_{\alpha_{i}}$ If $e_{\alpha_{i}} \neq e_{\alpha_{i+1}}$ then, since $U_{\alpha_{i}}$ corresponds to $R e_{\alpha_{i}}$ under the equivalence $\operatorname{Hom}_{\Delta}(U,-): \operatorname{Add}\left({ }_{\Delta} U\right) \rightarrow \operatorname{Proj}\left({ }_{R} R\right), R e_{\alpha_{i}} \neq R e_{\alpha_{i+1}}$ and, hence, $a_{i} \in e_{\alpha_{i}} R e_{\alpha_{i+1}} \subseteq e_{\alpha_{i}} J e_{\alpha_{i+1}}$. Thus, since $J$ is left $T$-nilpotent, there is an $n$ such that $a_{0} a_{1} \cdots a_{n}=0$.

(d) $\Rightarrow$ (c). Assume (d). Let ${ }_{\Delta} M$ be finitely generated and indecomposable. Let $s \in \operatorname{End}\left({ }_{\Delta} M\right)$. Then the noetherian condition implies that $s$ is either invertible or nilpotent, so $\operatorname{End}\left({ }_{\Delta} M\right)$ is local. In particular, $\Delta=\Delta g_{1} \oplus \cdots$ $\oplus \Delta g_{n}$ with the $g_{i}$ local orthogonal idempotents, so $\Delta$ is semiperfect. This implies that each of the finitely generated $U_{\alpha}$ is a direct sum of indecomposable modules which, as we have just seen, must have local endomorphism rings. Thus we can find a complete set of local idempotents $\left(f_{\alpha}\right)_{\alpha \in A}$ for $R$ (here each 
$e_{\alpha}$ is a sum of some of the $f_{\alpha}^{\prime}$ 's). If $a_{0}, a_{1}, \ldots$ is a sequence in $J(R)$ then there exist idempotents $f_{0}, f_{1}, f_{2}, \ldots$ (each a sum of $f_{\alpha}$ 's) with

$$
a_{0} a_{1} \cdots a_{n}=f_{0} a_{0} f_{1} a_{1} f_{2} \cdots f_{n} a_{n} f_{n+1} .
$$

If none of these products is zero we can apply the König graph theorem to obtain a sequence $f_{\alpha_{0}}, f_{\alpha_{1}}, \ldots$ with none of the products

$$
f_{\alpha_{0}} a_{0} f_{\alpha_{1}} a_{1} \cdots f_{\alpha_{n}} a_{n} f_{\alpha_{n+1}}=0 .
$$

But this is contrary to the noetherian assumption because, since they belong to $J(R)$, none of the $f_{\alpha_{i}} a_{i} f_{\alpha_{i+1}}: U f_{\alpha_{i}} \rightarrow U f_{\alpha_{i+1}}$ is invertible.

(a) and (c) $\Rightarrow$ (e). If (a) holds then ${ }_{\Delta} \mathfrak{R}=\operatorname{Add}\left({ }_{\Delta} U\right) \approx \operatorname{Proj}\left({ }_{R} R\right)$. Since the argument $(\mathrm{a}) \Rightarrow(\mathrm{b})$ in [1, Theorem 6] clearly is valid for a ring with enough idempotents, each module in $\operatorname{Proj}\left({ }_{R} R\right.$ ) (and hence in ${ }_{\Delta} \mathfrak{T}$ ) has a decomposition that complements direct summands if $R$ is left perfect.

$(e) \Rightarrow(d)$. This is by [15, Lemma 9].

From this Theorem and Auslander's theorem [5] that an artin algebra whose left modules are direct sums of finitely generated modules is one of finite module type, we have (with necessity due to Tachikawa [17]) the

COROLlaRY . If $\Delta$ is finitely generated over its center, then $\Delta$ is of finite module type if and only if each left $\Delta$-rodule has a decomposition that complements direct summands.

Together with [9], the Theorem yields the following result (which was already known to Auslander and to Gruson and Jensen).

COROLLARY . If every left and every right $\Delta$-module is a direct sum of finitely generated modules then $\Delta$ is a ring of finite module type.

REMARK. (1) Let $\left(V_{\beta}\right)_{\beta \in B}$ represent one copy of each finitely generated indecomposable left $\Delta$-module. If we make the additional assumption that $1 \in \Delta$ is a sum of orthogonal primitive idempotents (so that $\oplus_{B} V_{\beta}$ is a generator) then we can replace $R$ in conditions (b) and (c) of the Theorem with the functor ring $R^{\prime}$ of the $\left(V_{\beta}\right)_{\beta \in B}$.

(2) "Every left $\Delta$-module" in condition (e) can be replaced by "every countably generated left $\Delta$-module" or by "every countable direct sum of finitely generated left $\Delta$-modules."

(3) In [2], [4] (and [16]) there has been established a 1-1 correspondence between the rings of finite module type and the Morita equivalence classes of artinian (semiprimary) rings that have global dimension $\leqslant 2$ and dominant dimension $\geqslant 2$. The correspondence is $\Delta \leftrightarrow R^{\prime}=\operatorname{End}\left(V_{1} \oplus \cdots \oplus V_{n}\right)$ where $V_{1}, \ldots, V_{n}$ represent the indecomposable left $\Delta$-modules. It seems likely, especially in view of the results of [12]-[14] that an appropriate extension of this correspondence might help to determine whether or not rings whose left modules are direct sums of finitely generated modules need be rings of finite module type. (Are they even right artinian?)

(4) Auslander [5] has actually shown that if every indecomposable left module over an artin algebra is finitely generated, the algebra is of finite 
module type. His proof of this theorem and the results of [4] show that the conditions: $\left(\mathrm{a}^{\prime}\right) \Delta$ is left artinian and every indecomposable left $\Delta$-module is finitely generated; and $\left(\mathrm{d}^{\prime}\right) \Delta$ is left artinian and has no infinite sequences $M_{0} \rightarrow M_{1} \rightarrow M_{2} \rightarrow \cdots$ of proper monomorphisms between finitely generated indecomposable left modules, also serve to characterize the rings of the title.

\section{REFERENCES}

1. F. W. Anderson and K. R. Fuller, Modules with decompositions that complement direct summands, J. Algebra 22 (1972), 24l-253. MR 46 \#209.

2. M. Auslander, Representation dimension of artin algebras, Queen Mary College Notes, London, 1971.

3. - Notes on representation theory of artin algebras (written by I. Reiten), Brandeis Univ., Waltham, Mass., 1971-1972.

4. - Representation theory of artin algebras. II, Comm. Algebra 1 (1974), 269-310.

5. - Large modules over artin algebras, (to appear).

6. S. U. Chase, Direct products of modules, Trans. Amer. Math. Soc. 97 (1960), 457-473. MR $22 \# 11017$.

7. D. Eisenbud and P. Griffith, The structure of serial rings, Pacific J. Math. 36 (1971), 109121. MR 45 \# 1968.

8. C. C. Faith and E. A. Walker, Direct-sum representations of injective modules, J. Algebra 5 (1967), 203-221. MR 34 \#7575.

9. K. R. Fuller and I. Reiten, Note on rings of finite representation type and decompositions of modules Proc. Amer. Math. Soc. 50 (1975), 92-94.

10. P. Gabriel, Des catégories abéliennes, Bull. Soc. Math. France 90 (1962), 323-448. MR 38 \#1144.

11. P. A. Griffith, On the decomposition of modules and generalized left uniserial rings, Math. Ann. 184 (1969/70), 300-308. MR 41 \# 1790.

12. M. Harada, Perfect categories. I, Osaka J. Math. 10 (1973), 329-341.

13. _-, Perfect categories. II, Osaka J. Math. 10 (1973), 343-355.

14. - Perfect categories. III, Osaka J. Math. 10 (1973), 357-367.

15. M. Harada and Y. Sai, On categories of indecomposable modules. I, Osaka J. Math. 7 (1970), 323-344. MR 44 \#4066.

16. C. M. Ringel and H. Tachikawa, QF-3 rings, J. Reine Angew. Math. 272 (1975), 49-72.

17. H. Tachikawa, QF-3 rings and categories of projective modules, J. Algebra 28 (1974), 408413.

18. R. B. Warfield, Jr., A Krull-Schmidt theorem for infinite sums of modules, Proc. Amer. Math. Soc. 22 (1969), 460-465. MR 39 \#213.

Department of Mathematics, University of Iowa, Iowa City, Iowa 52242 